\title{
CURVES INVARIANT UNDER POINT-TRANSFORMATIONS OF SPECIAL TYPE*
}

BY

MARY F. CURTIS

Introduction. The analytic curves which are invariant under an analytic point-transformation with a fixed point and go through the fixed point have been elegantly treated by Lattès, $\dagger$ who showed that for a transformation of a certain general class there exist just two invariant analytic curves. The facts for the transformations which Lattès' methods can not handle are more complicated. In the particular transformations investigated in this paper, of importance because of their relation to the problem of three bodies $\neq$, it turns out that there may be no formally invariant curve, a finite number or a single infinity and that, even when a formally invariant series development exists, it is not, as in Lattès' case, always convergent.

1. Preliminary discussion. Given the point transformation:

$$
Q: \quad x_{1}=X(x, y), y_{1}=Y(x, y),
$$

where at least one point, taken without loss of generality as $(0,0)$, remains fixed and where the functions:

$$
X(x, y)=\sum_{i+j=1}^{\infty} a_{i j} x^{i} y^{j}, \quad Y(x, y)=\sum_{i+j=1}^{\infty} b_{i j} \dot{x}^{i} y^{j}
$$

are single-valued, analytic functions of the complex variables $x, y$ in $(0,0)$. We fix our attention on those transformations for which the roots $\rho_{1}, \rho_{2}$ of the characteristic equation: $\S$

$$
\left|\begin{array}{cr}
X_{x}-\rho & X_{y} \\
Y_{x} & Y_{y}-\rho
\end{array}\right|_{(0,0)}=0
$$

* Presented to the Society, September 5, 1918.

$\dagger$ Lattès, Sur les équations fonctionelles qui définissent une courbe ou une surface invariante par une transformation, A n na $1 \mathrm{i}$ d i M a t e m a t i ¿ a, ser. 3, vol. 13 (1906-1907), pp. 1-137.

† Levi-Civita, Com p tes R e nd u s, vol. 131, p. 103; Sopra alcuni criteri di instabilitd, A n n a $1 \mathrm{i}$ d i M a t e m a t i c a, ser. 3, vol. 5 (1901), pp. 221-307.

$\S$ Picard, Traité d'Analyse, vol. 3, pp. 23-46; Poincaré, Sur les courbes définies par les équations différentielles, 4e Partie, J o u n a 1 d e L i o u vile, (1886), pp. 173-195; Lattès, loc. cit. 
are both equal to unity* and for which not all the coefficients of the quadratic terms are zero, namely the transformations:

(2) $T$ :

$$
\begin{aligned}
& x_{1}=x+\mu(x, y)=x+\sum_{i+j=2}^{\infty} a_{i j} x^{i} y^{j}, \\
& y_{1}=y+\nu(x, y)=y+\sum_{i+j=2}^{\infty} b_{i j} x^{i} y^{j}
\end{aligned}
$$

where the $a_{i j}, b_{i j}, i+j=2$, are not all zero. We propose to determine conditions for the existence of analytic curves through $(0,0)$ invariant under (2) restricting the discussion to those curves for which $(0,0)$ is a regular point. $\dagger$

If the curve $y=\psi(x)$ :

$$
\psi(x)=\sum_{i=1}^{\infty} l_{i} x^{i}, \text { analytic at } x=0
$$

is invariant under the transformation $T$, given by (2), that is, if $y_{1}=\psi\left(x_{1}\right)$ transformed by $T^{-1}$ yields $y=\psi(x)$, we have

$$
\psi(x)+\nu(x, \psi(x))=\psi(x+\mu(x, \psi(x))) .
$$

By equating coefficients of corresponding powers of $x$ on the two sides of this identity we obtain a set of relations in the coefficients $l_{i}$ of $\psi(x)$ and in the coefficients $a_{i j}, b_{i j}$ of (2). These relations constitute a set of necessary conditions on the $l_{i}$ for the existence of an analytic curve or curves $y=\psi(x)$ invariant under (2). Moreover the sets of solutions $l_{1}, l_{2}, \ldots$ of these relations determine all the developments (3) formally invariant under (2), that is, determine all the formally invariant curves.

* The general transformations (1), as far as the linear terms are concerned, fall into three least invariant classes with respect to the group of affine transformations $S$, of $\$ 3$, leaving the origin fixed. If the roots $\rho_{1}, \rho_{2}$ are distinct, there exists an $S$ such that the transform of $Q$ by $S$ is of the form:

$$
x_{1}=\rho_{1} x+\sum_{i+j=2}^{\infty} a_{i j} x^{i} y^{j}, \quad y_{1}=\rho_{2} y+\sum_{i+j=2}^{\infty} b_{i j} x^{i} y^{j}, \quad \rho_{1} \rho_{2} \neq 0 .
$$

If $\rho_{1}=\rho_{2}=\rho \neq 0$, there exists an $S$, transforming (1) into

$$
x_{1}=\rho x+y+\sum_{i+j=2}^{\infty} a_{i j} x^{i} y^{j}, \quad y_{1}=\rho y+\sum_{i+j=2}^{\infty} b_{i j} x^{i} y^{j}, \rho \neq 0,
$$

except when (1) is already of the form:

$$
x_{1}=\rho x+\sum_{i+j=2}^{\infty} a_{i j} x^{i} y^{j}, \quad y_{1}=\rho y+\sum_{i+j=2}^{\infty} b_{i j} x^{i} y^{j}, \rho \neq 0 .
$$

The transformations $(1 a)$ have been completely investigated by Lattès except for the cases in which $\rho_{1}=\rho_{2}{ }^{n}, \rho_{2}=\rho_{1}{ }^{n}, n$ a positive integer.

$\dagger$ Study: Ebene analytische Kurven, 1911, p. 39. 
The first four relations obtained from (4), aside from the trivial relation $l_{1}$ $=l_{1}$, are:

$$
\begin{aligned}
& l_{1} \alpha_{2}-\beta_{2}=0 \\
& l_{1} \alpha_{3}-\beta_{3}+l_{2}\left(2 \alpha_{2}+\alpha_{2}^{\prime} l_{1}-\beta_{2}^{\prime}\right)=0 \\
& l_{1} \alpha_{4}-\beta_{4}+l_{3}\left(3 \alpha_{2}+\alpha_{2}^{\prime} l_{1}-\beta_{2}^{\prime}\right)+l_{2}^{2}\left(2 \alpha_{2}^{\prime}+\frac{1}{2}\left(\alpha_{2}^{\prime \prime} l_{1}-\beta_{2}^{\prime \prime}\right)\right) \\
& \quad+l_{2}\left(\alpha_{2}^{2}+2 \alpha_{3}+\alpha_{3}^{\prime} l_{1}-\beta_{3}^{\prime}\right)=0 \\
& \begin{array}{r}
l_{1} \alpha_{5}-\beta_{5}+l_{4}\left(4 \alpha_{2}+\alpha_{2}^{\prime} l_{1}-\beta_{2}^{\prime}\right)+l_{2} l_{3}\left(5 \alpha_{2}^{\prime}+\alpha_{2}^{\prime \prime} l_{1}-\beta_{2}^{\prime \prime}\right)+l_{2}^{3} \alpha_{2}^{\prime \prime} \\
+l_{3}\left(3 \alpha_{3}+3 \alpha_{2}^{2}+\alpha_{3}^{\prime} l_{1}-\beta_{3}^{\prime}\right)+l_{2}^{2}\left(2 \alpha_{3}^{\prime}+2 \alpha_{2} \alpha_{2}^{\prime}+\frac{1}{2}\left(\alpha_{3}^{\prime \prime} l_{1}-\beta_{3}^{\prime \prime}\right)\right) \\
+l_{2}\left(2 \alpha_{4}+2 \alpha_{2} \alpha_{3}+\alpha_{4}^{\prime} l_{1}-\beta_{4}^{\prime}\right)=0,
\end{array}
\end{aligned}
$$

where the primes denote differentiation with respect to $l_{1}$ and

$$
\alpha_{i}=\sum_{r+s=i} a_{r s} l_{1}^{s}, \quad \beta_{i}=\sum_{r+s=i} b_{r s} l_{1}^{s} .
$$

The compact formula for the $n$th relation obtained from (4) is derived in $\S 2$. The derivation is complicated and unessential for reading what follows. It is sufficient to note that the formula furnishes an immediate proof that the $n$th relation contains no $l$ with subscript greater than $n$ and that it involves $l_{n}$ linearly with the coefficient:

$$
\begin{aligned}
\lambda_{n}\left(l_{1}\right)=n \alpha_{2}+\alpha_{2}^{\prime} l_{1}-\beta_{2}^{\prime}=(n+2) a_{02} l_{1}^{2}+\left((n+1) a_{11}-2 b_{02}\right) l_{1} & \\
& +n a_{20}-b_{11}, n>1 .
\end{aligned}
$$

The first equation in (I) is a cubic in $l_{1}$ with coefficients from the quadratic terms of (2). If we set $\omega_{1}=a_{02}, 3 \omega_{2}=a_{11}-b_{02}, 3 \omega_{3}=a_{20}-b_{11}, \omega_{4}=-b_{20}$, it may be written:

$$
L\left(l_{1}\right)=\omega_{1} l_{1}^{3}+3 \omega_{2} l_{1}^{2}+3 \omega_{3} l_{1}+\omega_{4}=0 .
$$

When the $\omega$ 's are not all zero (6) has at most three distinct roots in $l_{1}$; there are then at the origin at most three possible slopes for an invariant curve $y=\psi(x)$. The directions determined by these slopes we shall call the invariant directions.

If a root $l_{1}=l_{11}$ of $(6)$ is substituted in the second relation in (I) and $\lambda_{2}\left(l_{11}\right) \neq 0$, $l_{2}$ is uniquely determined: $l_{2}=l_{21}$; if this process is continued and always $\lambda_{n}\left(l_{11}\right)$ $\neq 0$, the coefficients $l_{i}$ in (3) are all uniquely determined.

THEOREM. If $l_{11}$ is a root of (6) and not a root of $\lambda_{n}\left(l_{1}\right)=0, n>1$, there is one and only one formally invariant curve tangent at the origin to the invariant direction of slope $l_{11}$. If, moreover, (6) has three distinct roots and no one of them satisfies $\lambda_{n}\left(l_{1}\right)=0, n>1$, there are precisely three formally invariant curves through the origin.

It may happen that $\lambda_{i}\left(l_{11}\right)=0$; if then the $i$ th relation is not illusory, $l_{1}=l_{11}$ leads to a contradiction; if the $i$ th relation is illusory, $l_{i}$ may be undetermined 
and there is a possibility of $\infty^{1}$ formally invariant curves all tangent at the origin to the invariant direction of slope $l_{11}$.

It is conceivable that $l_{11}, l_{12}, l_{13}$ might all lead to contradictions. We shall show in $\$ 4$, however, that when (6) has three distinct roots there is always one which yields a formally invariant curve. A simple root of (6) can, as we shall prove, satisfy at most one equation $\lambda_{n}\left(l_{1}\right)=0$, while a multiple root either satisfies no equation $\lambda_{n}\left(l_{1}\right)=0$ and hence yields a single formal $\psi$-series, or it satisfies every equation $\lambda_{n}\left(l_{1}\right)=0$ and various possibilities arise. If the cubic (6) is illusory, $\lambda_{n}\left(l_{1}\right)=0$ is satisfied by at most one value of $l_{1}$ and this is the same for every $n$; hence we may say that for "almost every value of $l_{1}$ " there is a formally invariant curve.

Some of the difficulties involved in proving a formally invariant series $\Sigma_{i=1}^{\infty} l_{i} x^{i}$ actually convergent are evident from the discussion in $\$ 6$ of a particular transformation, for which a formally invariant series always exists but is convergent only when very special conditions are fulfilled.

2. The general formula for the determination of the coefficients in the power series development of an invariant curve. In order to write in compact form the general relation (I), we introduce a special notation. Given $t_{1}, t_{2}, t_{3}, \ldots$, where we assume that the weight of $t_{n}$ is $n-1$ and that the weight of a product in the $t^{\prime}$ s is the sum of the weights of the factors; then by $S_{j, k}\left(t_{i}, t_{i+1}, \ldots\right)$, $i \geqq 1$, we mean the sum of all different ordered products $t_{m_{1}} t_{m_{2}} \ldots t_{m j}$ in $t_{i}, t_{i+1}, \ldots$ which are of degree $j, j$ a positive integer, and of weight $k, k$ a positive integer or zero:

$$
S_{j, k}\left(t_{i}, t_{i+1}, \ldots\right)=\sum t_{m_{1}+m_{2}+\ldots+m_{j}=j+k} t_{m_{2}} \ldots t_{m_{j}}
$$

where the summation is over all possible different ordered sets: $m_{1}, m_{2}, \ldots$, $m_{j}$, such that $m_{n} \geqq i$ and $\Sigma_{n=1}^{j} m_{n}=j+k$. If $i>k / j+1$, there exist no products $t_{m_{1}} \ldots t_{m_{j}}$ as defined:

$$
S_{j, k}\left(t_{i}, t_{i+1}, \ldots\right)=0(i>k / j+1)
$$

in case $i \leqq k / j+1$, products $t_{m_{1}} \ldots t_{m_{j}}$ as defined do exist and for them $i \leqq m_{n} \leqq k-i j+i+j$, that is,

$$
S_{j, k}\left(t_{i}, \ldots\right)=S_{j, k}\left(t_{i}, \ldots, t_{p}\right)(i \leqq k / j+1, p=k-i j+i+j) .
$$

We also agree that

$$
S_{0,0}\left(t_{i} t_{i+1} \ldots\right)=1, S_{0, k}\left(t_{i}, t_{i+1}, \ldots\right)=0, k \neq 0 \text { for all } i .
$$


If $y=\Sigma_{i=1}^{\alpha} l_{i} x^{i}$, then the coefficient of $x^{j+n}$ in $y^{j}, j \geqq 0$, is $S_{j, n}\left(l_{1}, \ldots, l_{n+1}\right)$. Putting $y^{j}=\Sigma_{n=0}^{\alpha} S_{j, n}\left(l_{1}, \ldots, l_{n+1}\right) x^{j+n}$ into $x_{1}=x+\Sigma_{i+j=2}^{\infty} a_{i j} x^{i} y^{j}$ and denoting the total coefficient of $x^{k}$ in the result by $A_{k}$, we have

$$
A_{1}=1, A_{k}=\sum_{i+j=2}^{k} a_{i j} S_{j, k-i-j}\left(l_{1}, \ldots, l_{k-i-j+1}\right) k>1 .
$$

The $j$ th power of $x_{1}: x_{1}^{j}=\left(\Sigma_{k=1}^{\infty} A_{k} x^{k}\right)^{j}$ is $\Sigma_{n=0}^{\infty} S_{j, n}\left(A_{1}, \ldots, A_{n+1}\right) x^{j+n}$; accordingly the total coefficient of $x^{k}$ on the right-hand side of (4) is

$$
\sum_{j=1}^{k} l_{j} S_{j, k-j}\left(A_{1}, \ldots, A_{k-j+1}\right)
$$

Since $S_{j, 0}\left(A_{1}\right)=A_{1}^{j}=1$, this becomes $l_{1}$ for $k=1$, and becomes

$$
l_{k}+\sum_{j=1}^{k-1} l_{j} S_{j, k-j}\left(A_{1}, \ldots, A_{k-j+1}\right) \text { for } k>1 .
$$

To get the coefficient of $x^{k}$ on the left-hand side of (4), we have

$$
\begin{gathered}
y_{1}=y+\sum_{i+j=2}^{\infty} b_{i j} x^{i} y^{j}=\sum_{k=1}^{\infty}\left(l_{k}+B_{k}\right) x^{k}, \text { where } \\
B_{1}=0, \quad B_{k}=\sum_{i+j=2}^{k} b_{i j} S_{j, k-i-j}\left(l_{1}, \ldots, l_{k-i-j+1}\right), \quad k>1 .
\end{gathered}
$$

The general relation (I) may therefore be written:

$$
\sum_{j=1}^{k-1} l_{j} S_{j, k-j}\left(A_{1}, \ldots, A_{k-j+1}\right)=B_{k}, k>1 .
$$

To aid in the expansion of $(7 a)$ we next show that

(II $a) \quad S_{j, n}\left(t_{1}, \ldots, t_{n+1}\right)=j ! \sum \frac{t_{1}^{j-i_{1}}}{\left(j-i_{1}\right) !} \frac{t_{2}^{i_{1}-i_{2}}}{\left(i_{1}-i_{2}\right) !} \frac{t_{2}^{i_{2}-i_{2}}}{\left(i_{2}-i_{3}\right) !} \cdots \frac{t_{n}^{i_{n-1}-i_{n}}}{\left(i_{n-1}-i_{n}\right) !} \frac{t_{n+1}^{i_{n}}}{i_{n} !}$

where $j \geqq i_{1} \geqq i_{2} \geqq \cdots \geqq i_{n}$ and $\Sigma_{k=1}^{n} i_{k}=n$. For, from the definition of $\mathrm{S}_{j, k}\left(t_{i}, \ldots\right)$

$$
\begin{gathered}
S_{j, n}\left(t_{1}, \ldots, t_{n+1}\right)=\sum_{i_{1}=0}^{j} \frac{j !}{\left(j-i_{1}\right) ! i_{1} !^{j-i_{1}} S_{i_{1}, n}\left(t_{2}, \ldots, t_{n-i_{1}+2}\right),} \\
S_{i_{1}, n}\left(t_{2}, \ldots, t_{n-i_{1}+2}\right)=\sum_{i_{2}=0}^{i_{1}} \frac{i_{1} !}{\left(i_{1}-i_{2}\right) ! i_{2} !} t_{2}^{i_{1}-i_{2}} S_{i_{2}, n-i_{1}+i_{2}}\left(t_{3}, \ldots, t_{n-i_{1}-i_{2}+3}\right)
\end{gathered}
$$


and so on; hence

$$
\begin{aligned}
S_{j, n}\left(t_{1}, \ldots, t_{n+1}\right)=j ! & \sum_{i=0}^{j} \frac{t_{1}^{j-i_{1}}}{\left(j-i_{1}\right) !} \sum_{i_{2}=0}^{i_{1}} \frac{t_{2}^{i_{1}-i_{2}}}{\left(i_{1}-i_{2}\right) !} \\
& \ldots, \sum_{i_{n}=0}^{i_{n}-1} \frac{t_{n}^{i_{n}-1}-i_{n}}{\left(i_{n-1}-i_{n}\right) !} \sum_{n+1}^{i_{n}}=0 \frac{t_{n+1}^{i_{n}-i_{n}+1}}{\left(i_{n}-i_{n+1}\right) !} S_{i_{n+1}}, q\left(t_{n+2}, \ldots\right)
\end{aligned}
$$

where $q=n-\Sigma_{k=1}^{n} i_{k}+n i_{n+1}$.

Since $S_{j, n}\left(t_{1}, \ldots, t_{n+1}\right)$ has no $t$ with subscript greater than $n+1, S\left(t_{n+2}, \ldots\right)$ is independent of $t$ and is equal to unity; hence both its degree and weight must be zero. Accordingly $i_{n+1}=0, n-\Sigma_{k=1}^{n} i_{k}=0$ and the last sum becomes $\frac{i_{n+1}^{i_{n}}}{i_{n} !}$. If we use a single summation sign, understanding thereby this, that the summation is taken over all possible ordered values of $i_{1}, \ldots, i_{n}$ for which $j \geqq i_{1} \geqq i_{2} \geqq \cdots \geqq i_{n}$ and $\Sigma_{k=1}^{n} i_{k}=n$, we have the formula (II $a$ ).

By means of (II $a$ ) and the formulas for $A_{k}$ and $B_{k}$ we could write out the relations $(7 a)$. We facilitate this, however, by rewriting, with the aid of (II $b$ ), the relations ( $7 a)$ and the formulas for $A_{k}$ and $B_{k}$ in forms showing the exact manner of their dependence on $l_{1}$. We have, namely, for $A_{k}$ and $B_{k}$, denoting differentiation with respect to $l_{1}$ by primes:

$$
\begin{aligned}
& A_{k}=\sum_{i=2}^{k} \sum_{j=0}^{m} \frac{1}{j !} \alpha_{i}^{[j]} S_{j, k-i}\left(l_{2}, \ldots, l_{k-i-j+2}\right), \quad(k>1, i \geqq 2), \\
& B_{k}=\sum_{i=2}^{k} \sum_{j=0}^{m} \frac{1}{j !} \beta_{i}^{[j]} S_{j, k-i}\left(l_{2}, \ldots, l_{k-i-j+2}\right) .
\end{aligned}
$$

where $m$ is the smaller of the two integers, $i, k-i$ and $\alpha_{i}=\sum_{r+s=i} a_{r s} l_{1}^{s}, \beta_{i}=\sum_{r+s=i} b_{r s} l_{1}^{s}$ Instead of $(7 a)$ we have:

$$
\sum_{i=1}^{m} \sum_{j=1}^{k-i} l_{j} \frac{j !}{(j-i) ! i !} S_{i, k-j}\left(A_{2}, \ldots, A_{k-i-j+2}\right)-B_{k}=0, k>1,
$$

where $m=k / 2$, if $k$ is even, and $m=(k-1) / 2$, if $k$ is odd. This is our final form for the relations for the determination of the $l$ 's. It may be expanded by means of (II $b)$ and by the formula: 


$$
S_{j, n}\left(t_{2}, \ldots, t_{n-j+2}\right)=j ! \sum \frac{t_{2}^{j-i_{2}}}{\left(j-i_{1}\right) !} \frac{t_{3}^{i_{1}-i_{2}}}{\left(i_{1}-i_{2}\right) !}
$$

$$
\ldots \frac{t_{n-j-1-i_{n-j}}^{i_{n-1}}}{\left(i_{n-j-1}-i_{n-j}\right) !} \frac{t_{n-j+2}^{i_{n-j}}}{i_{n-j} !}
$$

where $j \geqq i_{1} \geqq i_{2} \geqq \cdots \geqq i_{n-j}$ and $\Sigma_{k=1}^{n-j} i_{k}=n-j$. This formula is the analogue of (II $a)$ when the $i$ of $S_{j, n}\left(t_{i}, \ldots\right)$ has been increased from one to two.

From (II $c$ ), the weight of $A_{k}$ or $B_{k}$ in the l's is $k-2$; therefore the weight of $S_{j, n}\left(A_{i}, \ldots\right)$ in the $l$ 's is $n-j$. The weight in the l's of the $i$ th term in $(7 b)$ is $(k-j-i)+(j-1)=k-i-1$ and this has its largest value $k-2$ when $i=1$. Hence $(7 b)$ contains no $l$ with subscript greater than $k-1$ and is, except for the case $k=2$, linear in $l_{k-1}$.

The coefficient in $(7 b)$ of $l_{k-1}, k>2$, which we denote by $\lambda_{k-1}\left(l_{1}\right)$, is $\lambda_{k-1}\left(l_{1}\right)$ $=l_{1} \alpha_{2}^{\prime}-\beta_{2}^{\prime}+(k-1) \alpha_{2}=(k-1) a_{20}-b_{11}+\cdot\left(k a_{11}-2 b_{02}\right) l_{1}+(k+1) a_{02} l_{1}^{2}$, $k>2$. The terms $l_{1} \alpha_{2}^{\prime}-\beta_{2}^{\prime}$ come from $l_{1} S_{1, k-1}\left(A_{2}, \ldots, A_{k}\right) \equiv l_{1} A_{k}$ and $B_{k}$; the term $(k-1) \alpha_{2}$ is from $(k-1) l_{k-1} S_{1,1}\left(A_{2}\right) \equiv(k-1) l_{k-1} A_{2}$. In $(7 b)$ the terms of weight zero are $l_{1} \alpha_{k}-\beta_{k}$; they come from $l_{1} S_{1, k-1}\left(A_{1}, \ldots, A_{k}\right) \equiv$ $l_{1} A_{k}$ and $B_{k}$.

3. Invariants and invariant systems of equations for the transformations $T$. An analytic curve through the origin invariant under $T$ is transformed by an analytic point transformation $U$ leaving the origin fixed:

$$
U: \quad \bar{x}=\sum_{i+j=1}^{\infty} k_{i j} x^{i} y^{j}, \quad \bar{y}=\sum_{i+j=1}^{\infty} l_{i j} x^{i} y^{j},
$$

into an analytic curve through the origin invariant under $T^{\prime}: T^{\prime}=U^{-1} T U$. Hence the study of any transformation $T^{\prime}$ equivalent to $T$ with respect to the transformations $U$ suffices for $T$ and for all transformations equivalent to $T$, that is, for the least invariant class of transformations with respect to the group $U$, to which $T$ belongs. The least invariant classes of transformations $T$, as far as quadratic terms are concerned, are the same with respect to the group of all analytic transformations $U$ as they are with respect to the group of affine transformations:

$$
S: \quad \bar{x}=k_{10} x+k_{01} y, \quad \bar{y}=l_{10} x+l_{01} y, \quad \Delta=k_{10} l_{01}-k_{01} l_{10} \neq 0 .
$$

The equations giving the values of the coefficients $\bar{a}_{i j}, \bar{b}_{i j}$ of the quadratic terms of the transiormation $T^{\prime}: T^{\prime}=S^{-1} T S$ in terms of $a_{20}, a_{11}, a_{02}, b_{20}, b_{11}, b_{02}$ constitute the so-called "group of parameters."* If we introduce instead of

* If the transformation $T$ is to be area-preserving, $\omega_{5}, \omega_{6}$ must vanish. This geometrical significance of $\omega_{5}, \omega_{6}$ was suggested by Birkhoff's paper, Stability in dynamics, read before the Society, September, 1918. 
$a_{20}, \ldots, b_{02}$ the following linear combinations of them:

$$
\begin{gathered}
\omega_{1}=a_{02}, \omega_{2}=\frac{a_{11}-b_{02}}{3}, \omega_{3}=\frac{a_{20}-b_{11}}{3}, \\
\omega_{4}=-b_{20}, \quad \omega_{5}=\frac{a_{11}+2 b_{02}}{3}, \omega_{6}=-\frac{b_{11}+2 a_{20}}{3}
\end{gathered}
$$

so that

$$
\begin{aligned}
& a_{20}=\omega_{3}-\omega_{6}, \quad a_{11}=\omega_{5}+2 \omega_{2}, a_{02}=\omega_{1}, \\
& b_{20}=-\omega_{4}, \quad b_{11}=-\left(2 \omega_{3}+\omega_{6}\right), \quad b_{02}=\omega_{5}-\omega_{2},
\end{aligned}
$$

the group of parameters becomes:

$$
\begin{aligned}
& \bar{\omega}_{1}=\frac{1}{\Delta^{2}}\left\{k_{10}^{3} \omega_{1}-3 k_{10}^{2} k_{01} \omega_{2}+3 k_{10} k_{01}^{2} \omega_{3}-k_{01}^{3} \omega_{4}\right\}, \\
& \bar{\omega}_{2}=\frac{1}{\Delta^{2}}\left\{k_{01}^{2}\left(l_{01} \omega_{4}-l_{10} \omega_{3}\right)+2 k_{31} k_{10}\left(l_{10} \omega_{2}-l_{01} \omega_{3}\right)+k_{10}^{2}\left(l_{01} \omega_{2}-l_{10} \omega_{1}\right)\right\}, \\
& \bar{\omega}_{3}=\frac{1}{\Delta^{2}}\left\{l_{10}^{2}\left(k_{10} \omega_{1}-k_{01} \omega_{2}\right)+2 l_{10} l_{01}\left(k_{01} \omega_{3}-k_{10} \omega_{2}\right)+l_{01}^{2}\left(k_{10} \omega_{3}-k_{01} \omega_{4}\right)\right\}, \\
& \bar{\omega}_{4}=\frac{1}{\Delta^{2}}\left\{l_{01}^{3} \omega_{4}-3 l_{01}^{2} l_{10} \omega_{3}+3 l_{01} l_{10}^{2} \omega_{2}-l_{10}^{3} \omega_{1}\right\}, \\
& \bar{\omega}_{5}=\frac{1}{\Delta}\left\{k_{10} \omega_{5}+k_{01} \omega_{6}\right\}, \\
& \bar{\omega}_{6}=\frac{1}{\Delta}\left\{l_{01} \omega_{6}+l_{10} \omega_{5}\right\} .
\end{aligned}
$$

The matrix of the infinitesimal transformations of this group, $G_{4}\left(x, y, \omega_{1}\right.$, $\left.\ldots, \omega_{6}\right)$, is essentially the matrix for obtaining the covariants of the binary cubic: $\omega_{1} y^{3}+3 \omega_{2} x y^{2}+3 \omega_{3} x^{2} y+\omega_{4} x^{3}$, where $y=\omega_{6}, x=\omega_{5}{ }^{*}$ The relative invariants of $G_{4}$ are:

$$
\begin{aligned}
& f_{1}=\omega_{1} \omega_{6}^{3}+3 \omega_{2} \omega_{5} \omega_{6}^{2}+3 \omega_{3} \omega_{5}^{2} \omega_{6}+\omega_{4} \omega_{5}^{3}, \\
& f_{2}=\left(\omega_{1} \omega_{3}-\omega_{2}^{2}\right) \omega_{6}^{2}+\left(\omega_{1} \omega_{4}-\omega_{2} \omega_{3}\right) \omega_{5} \omega_{6}+\left(\omega_{2} \omega_{4}-\omega_{3}^{2}\right) \omega_{5}^{2}, \\
& f_{3}=6 \omega_{1} \omega_{2} \omega_{3} \omega_{4}-4 \omega_{1} \omega_{3}^{3}-4 \omega_{2}^{3} \omega_{4}-\omega_{1}^{2} \omega_{4}^{2}+3 \omega_{2}^{2} \omega_{3}^{2}
\end{aligned}
$$

that is, the binary cubic, its hessian and discriminant. The invariant systems of equations for $G_{4}$ are found from the matrix in the usual fashion.

The absolute invariants and invariant systems of equations determine the least invariant classes of transformations $T$, as far as the quadratic terms are concerned. If we denote by $\left(a_{20}, \ldots, b_{02}\right)$ the homogeneous coördinates of a

* I,ie, Continuierliche Gruppen, p. 733. 
point in five-dimensional space, these least invariant classes of transformations $T$ are represented by the following least invariant spreads in $R_{5}$ :

$$
\begin{array}{ll}
m_{3}: & f_{1}: f_{2}: f_{3}=a: b: c, a, b, c \text { not all zero; } \\
m_{2}: \quad \begin{cases}\Omega_{1}=\omega_{2} \omega_{6}^{2}+2 \omega_{3} \omega_{5} \omega_{6}+\omega_{4} \omega_{5}^{2}, & \omega_{5}, \omega_{6} \text { not both zero, } \\
\Omega_{2}=\omega_{1} \omega_{6}^{2}+2 \omega_{2} \omega_{5} \omega_{6}+\omega_{3} \omega_{5}^{2}, & \omega_{1}, \omega_{2}, \omega_{3}, \omega_{4} \text { not all zero; } \\
\omega_{2} \omega_{6}+\omega_{3} \omega_{5}=c \omega_{5} \omega_{6}, & c \text { arbitrary; }\end{cases} \\
m_{2}^{\prime}: \quad \omega_{5}=\omega_{6}=0, f_{3}=0, \text { rank of }\left\|\begin{array}{cc}
\omega_{1} \omega_{2} \omega_{3} \\
\omega_{2} \omega_{3} \omega_{4}
\end{array}\right\| \text { two; } \\
m_{1}: & \omega_{1}=\omega_{2}=\omega_{3}=\omega_{4}=0, \omega_{5}, \omega_{6} \text { not both zero; } \\
m_{1}^{\prime}: & \omega_{5}=\omega_{6}=0, \quad \text { rank of }\left\|\begin{array}{l}
\omega_{1} \omega_{2} \omega_{3} \\
\omega_{2} \omega_{3} \omega_{4}
\end{array}\right\| \text { one. }
\end{array}
$$

These classes remain mutually exclusive, though not necessarily least invariant when all terms of $T$ are used.

4. The existence of formally invariant curves. A sufficient condition that there exist along the invariant direction determined by a root of the cubic (6):

$$
L\left(l_{1}\right)=\omega_{1} l_{1}^{3}+3 \omega_{2} l_{1}^{2}+3 \omega_{3} l_{1}+\omega_{4}=0, \omega_{1}, \omega_{2}, \omega_{3}, \omega_{4} \text { not all zero, }
$$

just one formally invariant curve is that the resultant of $L\left(l_{1}\right)$ and $\lambda_{n}\left(l_{1}\right)$

$$
\lambda_{n}\left(l_{1}\right)=\frac{n+2}{3} L^{\prime}\left(l_{1}\right)+(n-1)\left(\omega_{5} l_{1}-\omega_{6}\right)=0 \quad(n=2,3, \ldots),
$$

does not vanish for $n>1, n$ an integer. This resultant set equal to zero and freed from a factor $\omega_{1}^{*}$ is:

$$
N(n)=(n-1)^{3} f_{1}+3(n+2)(n-1) f_{2}+(n+2)^{2} f_{3}=0 .
$$

THEOREM 1. A sufficient condition that there is in each of the invariant directions determined by the roots of (6) just one formally invariant curve is that (9) is satisfied by no integer greater than unity.

In determining whether a particular root of (6) satisfies an equation (8) it is essential to distinguish between simple and multiple roots of (6).

THEOREM 2. A simple root of (6) can satisfy at most one equation (8). A multiple root of (6) satisfies no equation (8) if $f_{1}, f_{2}, f_{3}$ are not all zero; it satisfies every equation (8), if $f_{1}=f_{2}=f_{3}=0$.

* If, for $T, \omega_{1}=0$, we have only to take an equivalent transformation whose representative point does not lie in $\omega_{1}=0$. This is always possible when $L\left(l_{1}\right) \not \equiv 0$. 
For, if $\lambda_{n_{1}}\left(\bar{l}_{1}\right)=0$ and $\lambda_{n_{2}}\left(\bar{l}_{1}\right)=0, n_{1} \neq n_{2}$, then $L^{\prime}\left(\bar{l}_{1}\right)=0, \omega_{5} \bar{l}_{1}-\omega_{6}=0$, and every equation (8) has $\bar{l}_{1}$ as a root.' If $\omega_{5} \bar{l}_{1}-\omega_{6}=0$ and $\omega_{5}=0$, then $\omega_{6}=0$ and $\bar{l}_{1}$ is one of the two roots of $L^{\prime}\left(l_{1}\right)=0$; if $\omega_{5} \neq 0, \bar{l}_{1}=\omega_{6} / \omega_{5}$ and this satisfies $L^{\prime}\left(l_{1}\right)=0$. In either case

$$
\Omega_{2}=\omega_{1} \omega_{6}^{2}+2 \omega_{2} \omega_{5} \omega_{6}+\omega_{3} \omega_{5}^{2}=0 .
$$

LEMMA 1. Two and hence all equations (8) have a common root when and only when $\Omega_{2}=0$. The common root always satisfies $L^{\prime}\left(l_{1}\right)=0$ and if $\omega_{5} \neq 0$, it is $\omega_{6} / \omega_{5}$.

A common root of the equations (8), if it is also a root of (6) is, since it satisfies $L^{\prime}\left(l_{1}\right)=0$, a multiple root of (6). In this case (9) is satisfied for all integral $n, n \geqq 2$, and hence $f_{1}=f_{2}=f_{3}=0$; conversely, if (6) has a multiple root and $f_{1}=f_{2}=f_{3}=0$, this multiple root satisfies every equation (8).

LEMMA 2. A root of (6) satisfies two and hence every equation (8) when and only when it is a multiple root of (6) and $f_{1}=f_{2}=f_{3}=0$.

A simple root of (6) can, therefore, satisfy at most one equation (8). On the other hand, if a multiple root of (6) satisfies one equation (8), then, since it satisfies $L^{\prime}\left(l_{1}\right)=0$, it satisfies every equation (8) and $f_{1}=f_{2}=f_{3}=0$. Hence, if $f_{1}, f_{2}, f_{3}$ are not all zero, a multiple root of (6) satisfies no equation (8). This completes the proof of Theorem 2 .

A simple root $\bar{l}_{1}$ of (6) either satisfies no equation (8) and hence yields a single formally invariant curve, or it satisfies just one equation $(8): \lambda_{m}\left(\overline{l_{1}}\right)=0$. If $\lambda_{m}\left(\overline{l_{1}}\right)=0$, then $\lambda^{n}\left(\overline{l_{1}}\right) \neq 0, n \neq m$, and $l_{2}, l_{3}, \ldots, l_{m-1}$ are uniquely determined from the first $m-1$ relations derived from (4). When their values: $\bar{l}_{1}, \bar{l}_{2}, \bar{l}_{3}, \ldots, \bar{l}_{m-1}$ are substituted in the $m$ th relation, it is either contradictory or illusory; if it is illusory, $l_{m}$ is undetermined and the subsequent relations give for any chosen $l_{m}$, since $\lambda_{n}\left(\overline{l_{1}}\right) \neq 0, n \neq m$, unique values of $l_{m+r}(r=1,2, \ldots)$.

THEOREM 3. A necessary and sufficient condition that a simple root of (6) yield a unique formally invariant curve is that it does not satisfy an equation (8). If it satisfies an equation (8), it yields either no formally invariant curve or a single infinity.

If $\bar{l}_{1}$ is a multiple root of (6) and $f_{1}=f_{2}=f_{3}=0$, there is by Theorem 2 an immediate contradiction unless the second relation of (I) : $\alpha_{3} l_{1}-\beta_{3}=0$ is satisfied by $\bar{l}_{1}$.

THEOREM 4. If $f_{1}, f_{2}, f_{3}$ are not all zero, a multiple root of (6) always gives a formally invariant curve. If $f_{1}=f_{2}=f_{3}=0$, a necessary condition that a multiple root of (6) lead to a formally invariant curve is that this root satisfy $\alpha_{3} l_{1}-\beta_{3}=0$.

We now proceed to determine when the conditions which we have set up for the existence of formally invariant curves are fulfilled. If $f_{1}, f_{2}, f_{3}$ are not all zero, there is always at least one formally invariant curve. This follows from 
Theorem 4 if (6) has a multiple root. To show that at least one of three distinct roots of (6) leads to a formally invariant curve, we shall prove a number of lemmas.

LEMмa 3. If $f_{1}, f_{2}, f_{3}$ are not all zero, then of the roots of (9) considered as an equation in $n$ :

$$
\left(f_{1}+3 f_{2}+f_{3}\right) n^{3}+3\left(-f_{1}+2 f_{3}\right) n^{2}+3\left(f_{1}-3 f_{2}+4 f_{3}\right) n-f_{1}+6 f_{2}+8 f_{3}=0,
$$

at most two can be integers greater than unity.

If $f_{1}, f_{2}, f_{3}$ are not all zero, (9) considered as an equation in $n$ is not illusory. If $f_{1}+3 f_{2}+f_{3}=0$ the lemma is obvious; if $f_{1}+3 f_{2}+f_{3} \neq 0$ and two of the roots are integers greater than unity: $r_{1} \geqq r_{2}>1$, the remaining root is $\left(r_{1}+r_{2}-2\right) /\left(r_{1} r_{2}-1\right)$ and hence is less than unity.

According to this lemma the three roots of (6) cannot each satisfy a different equation (8). That two of them can not satisfy $\lambda_{n}\left(l_{1}\right)=0, n>1$, while a third satisfies $\lambda_{n}\left(l_{1}\right)=0, m>1, m \neq n$, we shall show by proving that the condition that $L\left(l_{1}\right)$ have $\lambda_{n}\left(l_{1}\right)$ as a factor is the same as the condition that (9) have a double root.

The condition that $L\left(l_{1}\right)$ contain $\lambda_{n}\left(l_{1}\right)$ as a factor is that the rank of the matrix

$$
\left\|\begin{array}{lll}
(n+2) \omega_{1} & 2(n+2) \omega_{2}+(n-1) \omega_{5} & (n+2) \omega_{3}-(n-1) \omega_{6} \\
(n+2) \omega_{2}-(n-1) \omega_{5} & 2(n+2) \omega_{3}+(n-1) \omega_{6} & (n+2) \omega_{4}
\end{array}\right\|
$$

is less than two, that is, that

$$
\begin{aligned}
& d_{1}=2(n+2)^{2} d_{23}+(n+2)(n-1) d_{34}+(n-1)^{2} \omega_{6}^{2}=0 \\
& d_{2}=(n+2)^{2} d_{13}+(n+2)(n-1) d_{24}-(n-1)^{2} \omega_{5} \omega_{6}=0 \\
& d_{3}=2(n+2)^{2} d_{12}+(n+2)(n-1) d_{14}+(n-1)^{2} \omega_{5}^{2}=0
\end{aligned}
$$

where $d_{i j}$ denotes the determinant made up of the $i$ th and $\jmath$ th columns of the matrix:

Then

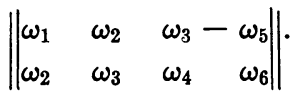

$$
\begin{aligned}
& \omega_{5}^{2} d_{1}+2 \omega_{5} \omega_{6} d_{2}+\omega_{6}^{2} d_{3}=2(n+2)^{2} f_{2}+(n+2)(n-1) f_{1}=0 \\
& d_{12} d_{1}-d_{13} d_{2}+d_{23} d_{3}=(n-1)^{2} f_{2}+(n+2)^{2} f_{3}=0 \\
& d_{14} d_{1}-2 d_{24} d_{2}+d_{34} d_{3}=2(n+2)(n-1) f_{2}+(n-1)^{2} f_{1}=0
\end{aligned}
$$

Hence

$$
f_{1}: f_{2}: f_{3}=2(n+2)^{3}:-(n-1)(n+2)^{2}:(n-1)^{3}, n>1
$$

Conversely, if $f_{1}: f_{2}: f_{3}$ are given by (11), it follows by retracing steps that the rank of the matrix (10) is less than two. 
LEMMA 4. A necessary and sufficient condition that two of the roots of (6) are the roots of $\lambda_{n}\left(l_{1}\right)=0, n>1$, is that

$$
f_{1}: f_{2}: f_{3}=2(n+2)^{3}:-(n-1)(n+2)^{2}:(n-1)^{3}, \quad n>1 \text {. }
$$

On the other hand, (9) has a double root, that is, $N(n)=0$ and $N^{\prime}(n)=0$ have a common root, when and only when $f_{1}: f_{2}: f_{3}$ are given by (11).

I.EMMA 5. A necessary and sufficient condition that two of the roots of (6) are the roots of $\lambda_{m}\left(l_{1}\right)=0, m>1$, is that $n=m$ is a double root of (9).

This completes the desired proof.

THEOREM 5. If $f_{1}, f_{2}, f_{3}$ are not all zero there exists one curve through the origin formally invariant under $T$.

If $f_{1}=f_{2}=f_{3}=0$, (6) always has a multiple root, which by Theorem 2 satisfies every equation (8) and, unless conditions depending on the coefficients of terms in $T$ of higher degree than the second are fulfilled, the multiple root yields no formally invariant curve. If $f_{2} \neq 0$, that is, if the rank of

$$
\left\|\begin{array}{lll}
\omega_{1} & \omega_{2} & \omega_{3} \\
\omega_{2} & \omega_{3} & \omega_{4}
\end{array}\right\|
$$

is two, (6) has also a simple root and this root yields in general a formally invariant curve. For, if the rank of (12) is two and $\omega_{5}=\omega_{6}=0$, the simple root $\bar{l}_{1}$ of (6) satisfies no equation (8). If $\omega_{5} \neq 0$, the double root is $\omega_{6} / \omega_{5}$, and the simple root is

$$
\overline{l_{1}}=-\frac{3 \omega_{2} \omega_{5}+2 \omega_{1} \omega_{6}}{\omega_{1} \omega_{5}}
$$

If $\lambda_{n}\left(\overline{l_{1}}\right)=0, n>1$, we have, using the notation of $(10 a)$

$$
n=\frac{\omega_{5}^{2}+2 d_{14}}{\omega_{5}^{2}-d_{14}}, n>1 \text {. }
$$

Similarly in case $\omega_{6} \neq 0$,

$$
n=\frac{\omega_{6}^{2}+2 d_{34}}{\omega_{6}^{2}-d_{34}}, n>1
$$

In place of either one of these ratios we may use the equal ratio:

$$
M=\frac{\omega_{5}^{2}+2\left(d_{14}+d_{34}\right)+\omega_{6}^{2}}{\omega_{5}^{2}-\left(d_{14}+d_{34}\right)+\omega_{6}^{2}},
$$

which can be shown to be an invariant when $f_{1}=f_{2}=f_{3}=0$ of the transformation $T$ under the affine transformations $S$. 
THEOREM 6. If $f_{1}=f_{2}=f_{3}=0$ and $f_{2} \not \equiv 0$, the simple root of (6) yields a formally invariant curve unless $M$ is defined and has a value equal to an integer greater than unity.

If (6) is illusory, $\lambda_{n}\left(l_{1}\right)=(n-1)\left(\omega_{5} l_{1}-\omega_{6}\right)$ and for every value of $l_{1}$ except perhaps for the value $\omega_{6} / \omega_{5}$ there exists a formally invariant curve.

5. Classification of the transformations $T$ according to the common factors of the quadratic terms. The least invariant classes of transformations $T$ as far as their quadratic terms:

$$
\alpha(x, y)=a_{20} x^{2}+a_{11} x y+a_{02} y^{2}, \beta(x, y)=b_{20} x^{2}+b_{11} x y+b_{02} y^{2},
$$

$\alpha(x, y), \beta(x, y)$ not both identically zero, are concerned, may be classified, on the basis fundamental in Levi-Civita's discussion of stability* into the three types:

TYPE 1. $\alpha(x, y),(\beta x, y)$ have no common factor in $x, y$;

TyPe 2. $\alpha(x, y), \beta(x, y)$ have one and only one common factor, linear in $x, y$;

TyPE 3. $\alpha(x, y), \beta(x, y)$ are linearly dependent.

In making the classification we assume $\omega_{4}=0 . \dagger \quad$ The least invariant spreads in this case are the intersections of the least invariant spreads $m_{3}, m_{2}, m_{2}^{\prime}, m_{1}$, $m_{1}^{\prime}$, of $G_{4}$ with $\omega_{4}=0$ :

$$
\begin{aligned}
& M_{2}: \quad f_{1}: f_{2}: f_{3}=a: b: c, a, b, c \text { not all zero; } \\
& M_{1}:\left\{\begin{array}{l}
\omega_{3}=\omega_{6}=0, \omega_{2}: \omega_{5}=g: h, g \neq 0 ; \\
D_{1}=D_{2}=D_{3}=0, \omega_{3}: \omega_{6}=g: h, g \neq 0 ;
\end{array}\right. \\
& M_{1}^{\prime}: \quad \omega_{2}=\omega_{3}=\omega_{6}=0, \omega_{1} \omega_{5} \neq 0 \text {; } \\
& M_{1}^{\prime \prime}: \quad \omega_{1}=\omega_{2}=\omega_{3}=0, \omega_{5}, \omega_{6} \text { not both zero; } \\
& M_{0}: \quad \omega_{2}=\omega_{3}=\omega_{5}=\omega_{6}=0, \omega_{1} \neq 0 \text {; }
\end{aligned}
$$

where $D_{i}$ denotes the determinant obtained from the matrix:

$$
\left\|\begin{array}{llr}
2 \omega_{1} & \omega_{2} & -\omega_{5} \\
3 \omega_{2} & 2 \omega_{3} & \omega_{6}
\end{array}\right\|
$$

by omitting the $i$ th column. In particular, $m_{3}, m_{1}, m_{1}^{\prime}$ yield, respectively, $M_{2}$, $M_{1}^{\prime \prime}, M_{0}$; the intersection of $m_{2}$ with $\omega_{4}=0$ consists of $M_{1}(g \neq 0)$ and $M_{1}^{\prime}$, while that of $m_{2}^{\prime}$ is $M_{1}(g=0)$. Each of the $\infty^{1}$ spreads $M_{1}$ is made up of two distinct continua as indicated; by the $G_{4}$ a point $P$ on one can be transformed into any

* Levi-Civita, A n n a 1 i d i M a t e m a t i c a, ser. 3, vol. 5 (1901), p. 242. He shows that the transformations:

$$
x_{1}=x+x^{2}+y(a x+b y)+U(x, y), y_{1}=y(1+c x+d y)+V(x, y)
$$

are unstable, except perhaps for $c=1$.

$\dagger$ If for a given $T, \omega_{4} \neq 0$, we have only to take an equivalent transformation for which $\omega_{4}=0$. 
point $P^{\prime}$ on the other, but by the sub-group $G_{3}$ with $l_{10}=0$, for which $\omega_{4}=0$ is an invariant equation, a point $P$ on one cannot be transformed into a point $P^{\prime}$ on the other.

The least invariant spreads for $\omega_{4}=0$ which are of Type 1 are those for which the resultant: $f_{1}+3 f_{2}+f_{3}$ of $\alpha(x, y)$ and $\beta(x, y)$ does not vanish. Those of Type 3 are determined by considering in detail the three cases: $\alpha(x, y) \neq \equiv 0$, $\beta(x, y) \not \equiv 0 ; \alpha(x, y) \not \equiv 0, \beta(x, y) \equiv 0 ; \alpha(x, y) \equiv 0, \beta(x, y) \not \equiv 0$. Those for Type 2 are then obtained by exclusion. The resulting classification of the least invariant spreads for $\omega_{4}=0$, together with a representative transformation for each least invariant spread, is:

\begin{tabular}{|c|c|c|}
\hline Type & Spread & Representative Transformations \\
\hline$(1 a)$ & $M_{2}(a+3 b+c \neq 0)$ & $\left\{x_{1}=x+a_{20} x^{2}+a_{11} x y+y^{2}+\cdots, a_{20} \neq 0\right.$ \\
\hline & & $\begin{cases}y_{1}=y & +x y\end{cases}$ \\
\hline$(2 a)$ & $\begin{array}{l}M_{2}(a+3 b+c=0, \\
a: b: c \neq 2:-1: 1)\end{array}$ & $\left\{\begin{array}{l}x_{1}=x+a_{11} x y+y^{2}+\cdots \\
y_{1}=y+x y+\cdots\end{array}\right.$ \\
\hline$(2 b)$ & $\begin{aligned} M_{1}(g: h \neq-1: 2 \\
g: h \neq 1: 1)\end{aligned}$ & $\left\{\begin{array}{l}x_{1}=x+a_{11} x y+\cdots, a_{11} \neq 0,1 \\
y_{1}=y+y^{2}+\cdots\end{array}\right.$ \\
\hline$(2 c)$ & $M_{1}^{\prime \prime}$ & $\left\{\begin{array}{l}x_{1}=x+x y+\cdots \\
y_{1}=y+y^{2}+\cdots\end{array}\right.$ \\
\hline$(2 d)$ & $M_{1}^{\prime}$ & $\left\{\begin{array}{l}x_{1}=x+x y+y^{2}+\cdots \\
y_{1}=y+y^{2}+\cdots\end{array}\right.$ \\
\hline$(3 a)$ & $M_{2}(a: b: c=2:-1: 1)$ & $\left\{\begin{array}{l}x_{1}=x+x y+\cdots \\
y_{1}=y+x y+\cdots\end{array}\right.$ \\
\hline$(3 b)$ & $M_{1}(g: h=-1: 2)$ & $\left\{\begin{array}{l}x_{1}=x+\cdots \\
y_{1}=y+y^{2}+\cdots\end{array}\right.$ \\
\hline$(3 c)$ & $M_{1}(g: h=1: 1)$ & $\left\{\begin{array}{l}x_{1}=x+x y+\cdots \\
y_{1}=y+\cdots\end{array}\right.$ \\
\hline$(3 d)$ & $M_{0}$ & $\left\{\begin{array}{l}x_{1}=x+y^{2}+\cdots \\
y_{1}=y+\cdots\end{array}\right.$ \\
\hline
\end{tabular}

The representative transformations as given for the non-singular least invariant spreads, that is, those for which $f_{1}, f_{2}, f_{3}$ are not all zero, are easily obtained by using the fact that when $\omega_{4}=0$ these non-singular least invariant spreads are given by:

$$
\begin{aligned}
& G=a \omega_{3}^{3}+3 b \omega_{3}^{2} \omega_{6}+c \omega_{6}^{3}=0 \\
& H=a \omega_{3}^{2}\left(\omega_{2}^{2}-\omega_{1} \omega_{3}\right)+b \omega_{1} \omega_{3}^{2} \omega_{6}-c \omega_{5} \omega_{6}\left(\omega_{2} \omega_{6}+\omega_{3} \omega_{5}\right)=0
\end{aligned}
$$

and by showing that it is always possible to take not only $b_{20}=0$ but also $b_{02}=0$, $b_{11}=1$. Those given for the singular least invariant spreads can be picked out at once. 
Each of these representative transformations has been chosen without regard to the particular invariant direction brought into coincidence with the axis of $x$. Hence for each representative transformation we have to consider the possibility of invariant curves not only in the direction of the $x$-axis but also in the other invariant directions. It is, however, of advantage to have the invariant direction to be discussed always along the $x$-axis, that is, to supplement each representative transformation by one or more transformations which have, respectively, along the $x$-axis the non-equivalent types of invariant directions for the least invariant spread in question. If the transformation $T$ as given has an invariant direction $l_{1}$, besides that along the $x$-axis, we transform $T$ into $\bar{T}$, so that $\bar{T}$ has its corresponding invariant direction $\bar{l}_{1}$, along the $x$-axis. If each $\bar{T}$ can be taken in the same form as $T$, then $T$ suffices; otherwise to $T$ must be added transformations representative of the $\bar{T}$ which can not be taken of the form $T$.

Denoting by the subscripts 1 and 2 two equivalent transformations with nonequivalent invariant directions along the $x$-axis, we have as our complete classification:

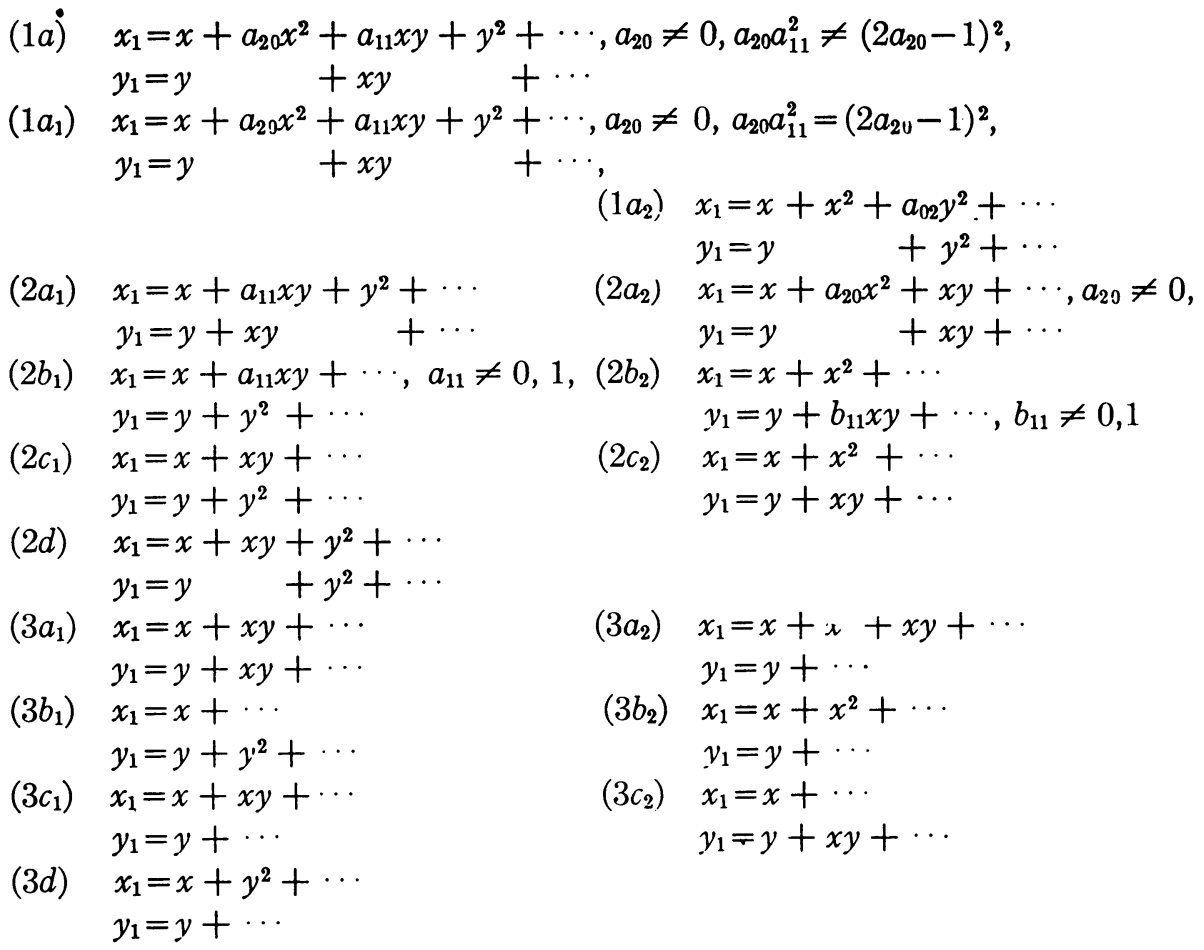

These type forms are mutually exclusive; moreover, two transformations of any type with different values for the constants of that type are mutually exclusive. 
From a consideration of equation (9) it follows that:

THEOREM: If the roots of (6) are distinct there are at least two curves formally invariant under Type $1 a_{i}$; if $a_{02}=0$ there are three. If the roots of (6) are distinct there are at least two curves formally invariant under Type $2 a_{i}$; if they are not distinct each root yields a formally invariant curve. There are under Type $3 a_{i}$ three formally invariant curves.

In Types $2 b_{2}, 2 c_{2}, 3 b_{2}, 3 c_{2}$ the simple invariant direction is along the $x$-axis and it is easy to discover, by computing the value of $M$, when the simple root of (6) gives trouble.

THEOREM. In Type $2 b_{2}$, if $b_{11}$ is not an integer greater than two, the simple root of (6) yields a formally invariant curve. In Type $2 c_{2}$, every root of (6) yields a formally invariant curve; hence there are under Type $2 c_{2} \infty^{1}$ formally invariant curves. In Types $3 b_{2}, 3 c_{2}$ the simple root of (6) yields a formally invariant curve.

6. Curves invariant under some special transformations. If the curve $y=\psi(x)$ is invariant under:

(13) $T$ :

$$
\begin{aligned}
& x_{1}=x+f(x)=\frac{x}{1-x}, \\
& y_{1}=y+\varphi(x), b_{2}=0,
\end{aligned}
$$

then $\psi(x)$ is a solution, analytic at the origin, of the functional equation:

$$
\psi(x)+\varphi(x)=\psi\left(\frac{x}{1-x}\right)
$$

The equations (I) here become $l_{1}=0$ and

$$
b_{k+1}=\frac{k !}{(k-1) !} l_{2}+\cdots+\frac{k !}{2 !(k-2) !} l_{k-1}+\frac{k !}{(k-1) !} l_{k} \quad(k=2,3, \ldots) .
$$

The equations $k=2, \ldots, k$ solved for $l_{k}$ give:

$$
\frac{1}{(k-1) !} l_{k}=\frac{1}{k !} b_{k+1}+M_{1} \frac{1}{(k-1) !} b_{k}+\cdots+M_{k-2} \frac{1}{2 !} b_{3} \quad(k=2,3, \ldots),
$$

where the $M$ 's satisfy the relations:

$$
\frac{1}{(k-1) !}+\frac{M_{1}}{(k-2) !}+\frac{M_{2}}{(k-3) !}+\cdots+M_{k-2}=0 \quad(k=3,4, \ldots),
$$


that is, are the coefficients in the power series development of the function $\frac{x}{e^{x}-1}$. Hence

$$
\begin{aligned}
\sum_{k=2}^{\infty} \frac{1}{(k-1) !} l_{k} \dot{x}^{k-1} & =\sum_{k=2}^{\infty}\left(\frac{1}{k !} b_{k+1}+M_{1} \frac{1}{(k-1) !} b_{k}+\cdots+M_{k-2} \frac{1}{2 !} b_{3}\right) x^{k} \\
& =\frac{x}{e^{x}-1} \frac{1}{x} \sum_{k=2}^{\infty} \frac{b_{k} x^{k-1}}{(k-1) !}
\end{aligned}
$$

If for an arbitrary series $\eta(x)=\Sigma_{n=2}^{\infty} v_{n} x^{n}$, we define the series $\eta_{1}(x)=\sum_{n=2}^{\infty} \frac{v_{n} x^{n-1}}{(n-1)}$ ! as the associated $\eta$-series, this relation becomes:

$$
\varphi_{1}(x)=\left(e^{x}-1\right) \psi_{1}(x)^{*}
$$

or since $f_{1}(x)=\sum_{n=2}^{\infty} \frac{x^{n-1}}{(n-1) !}=e^{x}-1$

$$
\varphi_{1}(x)=f_{1}(x) \psi_{1}(x)
$$

In order to determine when, for $f(x)=\Sigma_{n=2}^{\infty} x^{n}$ and a given $\varphi(x)$. convergent for $|x|<\rho$, there exists a convergent $\psi(x)$ satisfying (14) we shall have to discuss in detail the relationship of a series $\eta(x)$ to its associated series with respect to convergence and divergence. Since the series $\Sigma_{n=2}^{\infty} v_{n} x^{n-1}$ converges and diverges for the same $x$ as $\eta(x)$, we may equally well consider its relationship to $\eta_{1}(x)$ or more simply, the relationship of $\theta(x)=\Sigma_{n=1}^{\infty} n ! u_{n} x^{n}$ and $\theta_{1}(x)=\Sigma_{n=1}^{\infty} u_{n} x^{n}$.

If $\theta(x)$ converges, $\theta_{1}(x)$ converges for all $x$ and is an integral function; if $\theta_{1}(x)$ is an integral function, we make use of the classification of integral functions according to their strength of convergence, or, more precisely according to their $\lambda-, \mu-, \nu$-indices $\dagger$ in order to determine the behavior of $\theta(x)$.

The $\lambda$-index of the integral function $\theta_{1}(x)$, whose zeros are: $c_{1}, c_{2}, \ldots,\left|c_{1}\right| \leqq\left|c_{2}\right|$ $\leqq \ldots$, where $x=0$, if present, has been excluded, is, if it exists, the number $\lambda \geqq 0$, such that, for every positive $\epsilon, \sum_{n=1}^{\infty} \frac{1}{\left|c_{n}\right|^{\lambda+\epsilon}}$ converges, whereas $\sum_{n=1}^{\infty} \frac{1}{\left|c_{n}\right|^{\lambda-\epsilon}}$ diverges.

The $\mu$-index of $\theta_{1}(x)=\Sigma_{n=1}^{\infty} u_{n} x^{n}$ is, if it exists, the number $\mu \geqq 0$, such that to every positive $\epsilon$

(a) $\left|u_{n}\right|<\frac{1}{n !^{\mu-a}}$ for all $n>m_{e}$

(b) $\left|u_{n}\right|>\frac{1}{n !^{\mu+e}}$ for some arbitrarily large $n$.

* The deduction of this functional equation is due to C. L. Bouton.

$\dagger$ Vivanti-Gutzmer, Eindeutige analytische Funktionen, pp. 228-230. 
The $\nu$-index of $\theta_{1}(x)$ is, if it exists, the number $\nu \geqq 0$, such that to every positive $\epsilon^{*}$

(a) $\left|\theta_{1}(x)\right|<e^{|x|^{\nu+\epsilon}}$ for all $|x|>r_{\epsilon}$,

(b) $\left|\theta_{1}(x)\right|>e^{|x|^{\nu-\epsilon}}$ for some arbitrarily large $x$.

In case an index does not exist, it is said to be infinite. The following theorems are dependent on the definition of the $\mu$-index:

THEOREM 1. If $\theta_{1}(x)$ has $\mu$-index infinite, $\theta(x)$ is an integral function with $\mu$-index infinite, and conversely.

THEOREM 2. If $\theta_{1}(x)$ has $\mu$-index equal to $\mu_{1}$, then

(a) if $\mu_{1}>1, \theta(x)$ is an integral function, with $\mu$-index equal to $\mu_{1}-1>0$;

(b) if $\mu_{1}<1, \theta(x)$ is divergent and

$$
\begin{aligned}
& \left|n ! u_{n}\right|<n !^{1-\mu_{1}+\epsilon}, n>m_{\epsilon} \\
& \left|n ! u_{n}\right|>n !^{1-\mu_{1}-\epsilon}, \text { for some arbitrarily large } n \text {, and conversely. }
\end{aligned}
$$

If $\mu_{1}=1$, then $\left|n ! u_{n}\right|<n !^{\epsilon}$ for all $n>m_{\epsilon},\left|n ! u_{n}\right|>n !^{-\epsilon}$ for some arbitrarily large $n$, and $\theta(x)$ may be an integral function with $\mu$-index equal to zero, or may be convergent for $|x|<\rho$, or divergent. 'To distinguish these three cases, we introduce, in connection with the $\nu$-index, which here is unity, since $\mu \nu=1, \dagger$ a subindex $\gamma, \ddagger$ and thus obtain a further classification of integral functions with $\mu$-index unity.

For $\theta_{1}(x)$ with $\mu=\nu=1$,

(a) the $\gamma$-subindex is infinite, if $\left\{\begin{array}{l}\left|\theta_{1}(x)\right|<e^{|x|^{1+\epsilon}} \text { for all }|x|>r_{\epsilon}, \\ \left|\theta_{1}(x)\right|>e^{\frac{|x|}{\epsilon}} \text { for some arbitrarily large } x ;\end{array}\right.$

(b) the $\gamma$-subindex is $\gamma \neq 0$, if $\left\{\begin{array}{l}\left|\theta_{1}(x)\right|<e^{(\gamma+\epsilon)|x|} \text { for all }|x|>r_{\epsilon}, \\ \left|\theta_{1}(x)\right|>e^{(\gamma-\epsilon)|x|} \text { for some arbitrarily large } x \text {; }\end{array}\right.$

(c) the $\gamma$-subindex is zero, if $\left\{\begin{array}{l}\left|\theta_{1}(x)\right|<e^{\epsilon|x|} \text { for all }|x|>r_{\epsilon}, \\ \left|\theta_{1}(x)\right|>e^{|x|^{1-\epsilon}} \text { for some arbitrarily large } x .\end{array}\right.$

Pringsheim $\S$ shows that these three pairs of inequalities on $\left|\theta_{1}(x)\right|$ lead respectively to the following three pairs of inequalities on $\left|u_{n}\right|$ and conversely:

* Pringsheim, Ganze Funktionen, $\mathrm{M}$ a $\mathrm{t}$ h e $\mathrm{m}$ a $\mathrm{t}$ i s c h e A n n a 1 e n, vol. 58 (1904). p. 262.

† Pringsheim, loc. cit., p. 279; Vivanti-Gutzmer, loc. cit, p. 267

$\ddagger$ Pringsheim, loc. cit., p. 264.

§ Pringsheim, loc. cit., pp. 278-279. 
(a)

$$
\begin{aligned}
& \left\{\begin{array}{l}
\left|u_{n}\right|<\frac{1}{n !^{1-\epsilon}} \text { for all } n>m_{\epsilon}, \\
\left|u_{n}\right|>\frac{1}{n ! \epsilon^{n}} \text { for some arbitrarily large } n ;
\end{array}\right. \\
& \left\{\begin{array}{l}
\left|u_{n}\right|<\frac{(\gamma+\epsilon)^{n}}{n !} \text { for all } n>m_{\epsilon}, \\
\left|u_{n}\right|>\frac{(\gamma-\epsilon)^{n}}{n !} \text { for some arbitrarily large } n ;
\end{array}\right. \\
& \left\{\begin{array}{l}
\left|u_{n}\right|<\frac{\epsilon^{n}}{n !} \text { for all } n>m_{\epsilon}, \\
\left|u_{n}\right|>\frac{1}{n !^{1+\epsilon}} \text { for some arbitrarily large } n .
\end{array}\right.
\end{aligned}
$$

The corresponding inequalities on the coefficients of $\theta(x)$ are:

(a)

$$
\begin{aligned}
& \left\{\begin{array}{l}
\left|n ! u_{n}\right|<n !^{\epsilon} \text { for all } n>m_{\epsilon}, \\
\left|n ! u_{n}\right|>\epsilon^{-n} \text { for some arbitrarily large } n ;
\end{array}\right. \\
& \left\{\begin{array}{l}
\left|n ! u_{n}\right|<(\gamma+\epsilon)^{n} \text { for all } n>m_{e} \\
\left|n ! u_{n}\right|>(\gamma-\epsilon)^{n} \text { for some arbitrarily large } n ;
\end{array}\right. \\
& \left\{\begin{array}{l}
\left|n ! u_{n}\right|<\epsilon^{n} \text { for all } n>m_{\epsilon}, \\
\left|n ! u_{n}\right|>n !^{-\epsilon} \text { for some arbitrarily large } n .
\end{array}\right.
\end{aligned}
$$

Hence we conclude the following theorem:

THEOREM 3. If $\theta_{1}(x)$ has $\mu$-index equal to unity, then

(a) if $\gamma$ is infinite, $\theta(x)$ diverges;

(b) if $\gamma$ is finite, not zero, $\theta(x)$ converges for $|x|<\frac{1}{\gamma}$;

(c) if $\gamma$ is zero, $\theta(x)$ is an integral function with $\mu$-index equal to zero, and conversely.

We are now in a position to discuss the conditions for a solution of the equation (14).

THEOREM 4. A necessary and sufficient condition that there exist a convergent $\psi(x)$ satisfying (14) and hence that there exist an analytic curve $y=\psi(x)$ invariant under (13) is that $\varphi_{1}(x)$ contain among its zeros the zeros: $2 n \pi i, \boldsymbol{v}=0, \pm 1, \pm 2, \ldots$, of $f_{1}(x)=e^{x}-1$. The radius of convergence of $\psi(x)$, when $\psi(x)$ converges, is at least as great as that of $\varphi(x)$. 
The condition is necessary, for if $\psi(x)$ is convergent, $\psi_{1}(x)$ is an integral function and $\varphi_{1}(x)$ must have among its zeros the zeros of $f_{1}(x)$.

It remains to show the condition sufficient. The function $f_{1}(x)$ has the zeros $2 n \pi i$, its $\lambda$-, $\mu$-, $\nu$ - indices and $\gamma$-subindex are all unity. Since $\varphi(x)$ converges, $\mu_{\varphi_{1}}$ is, by Theorems 1 and 2 , greater than one, or, by the Theorem 3 , equal to one with $\gamma_{\varphi_{1}}$ finite. Suppose $\mu_{\varphi_{1}}>1$; then since $\nu=1 / \mu$ and $\lambda \geqq \nu^{*}$ it follows that $\lambda_{\varphi_{1}}<1$; but in order that $\varphi_{1}(x)$ have among its zeros the values $2 n \pi \mathrm{i}, \lambda_{\varphi_{1}}$ $\geqq 1$ and hence a contradiction. We thus have

$$
\mu_{\varphi_{1}}=1, \nu_{\varphi_{1}}=1 \text { and } \gamma_{\varphi_{1}} \text { finite, } \lambda_{\varphi_{1}}=1 \text {. }
$$

We next prove the lemma:

LEMMA: If for a given $\gamma, \nu,\left|\psi_{1}(x)\right|>e^{\gamma|x| \nu}$ for some arbitrarily large $x$, then for any positive $\epsilon$, there exists some arbitrarily large $x$ for which $\left|\varphi_{1}(x)\right|>e^{(\gamma-\epsilon)|x|^{\nu}}$.

With the points $x=2 n \pi i$ as centers and radius $\eta<\pi / 2$ draw circles. Denote by $S_{\eta}$ that part of the plane which remains after the interiors of the circles have been removed. In $S_{\eta},\left|e^{x}-1\right| \geqq\left(1-1 / e^{\eta}\right)$, for in the fundamental region: $-\pi \leqq x_{2}<\pi, x=x_{1}+i x_{2}$, exclusive of the interior of the circle $|x|=\eta, e^{x}-1$ has its minimum in $x=-\eta$.

Denote by $X$ those of the given $x$ which are not in $S_{\eta}$. We replace each $X$ by that $x$ in $S_{\eta}$ which lies on the circle, center in $X$, radius $2 \eta$ and in which $\left|\psi_{1}(x)\right|$ for the circle takes on its maximum value. Then by Cauchy's Abschätzung $\dagger$ we have

$\left|\psi_{1}(x)\right|>\left|\psi_{1}(X)\right|$, that is, $\left|\psi_{1}(x)\right|>e^{\gamma|X|^{\nu}}$. Since $|X|-|x| \geqq-2 \eta$, we have

$$
\left|\psi_{1}(x)\right|>e^{\gamma|x| \nu\left(1-\frac{2 \eta}{|x|}\right)^{\nu}}
$$

For each $\epsilon_{1}>0$ there exists $r_{e_{1}}$ such that $\left(1-\frac{2 \eta}{|x|}\right)^{\nu}>1-\frac{\epsilon_{1}}{\gamma}$, for $|x|>r_{\mathrm{e} 1}$.

Hence the relation

$$
\left|\psi_{1}(x)\right|>e^{\left(\gamma-\epsilon_{1}\right)|x|^{\nu}}
$$

holds for those $|x|>r_{\epsilon_{1}}$ in $S_{\eta}$ which replace the given $X$ not in $S_{\eta}$; it certainly holds for those of the given $x$ in $S_{\eta}$ and therefore holds for infinitely many arbitrarily large $x,|x|>r_{e_{1}}$. We accordingly have $\left|\varphi_{1}(x)\right|>e^{\left(\gamma-e_{1}\right)|x|^{\nu}}\left(1-1 / e^{n}\right)$ for some arbitrarily large $x,|x|>r_{\mathrm{el}}$. Given a positive $\epsilon$, take $\epsilon_{1}<\epsilon$; then there exists $r_{e} \geqq r_{e 1}$ such that $\left(1-1 / e^{n}\right)>e^{\left(e_{1}-e\right)|x|^{n}}$ for $|x|>r_{e}$ and hence $\left|\varphi_{1}(x)\right|$ $>e^{(\gamma-e)|x|^{\nu}}$ for some arbitrarily large $x,|x|>r_{\epsilon}$.

* Vivanti-Gutzmer, loc. cit., p. 238.

† Osgood, Funktionentheorie, 1st. edition, p. 255. 
We conclude at once that $\nu_{\psi_{1}}$ is finite, for if $\nu_{\psi_{1}}$ were infinite then, by the lemma, $\nu_{\varphi 1}$ would be infinite, whereas it is actually unity. Also by the lemma $\nu_{\psi_{1}} \leqq \nu_{\varphi_{1}}$, that is, $\nu_{\psi_{1}} \leqq 1, \mu_{\psi_{1}} \geqq 1$.

If $\mu_{\psi_{1}}>1$, by Theorem $2, \psi(x)$ converges for all $x$ and the sufficiency of the condition in Theorem 4 is in this case proved.

If $\mu_{\psi_{1}}=1$, then $\nu_{\psi_{1}}=\nu_{\varphi_{1}}=1$ and $\gamma_{\psi_{1}}$ is finite, for, if $\nu_{\psi_{1}}$ were infinite, the lemma applied for $\nu=1$ yields $\gamma_{\varphi 1}$ infinite which means that $\varphi(x)$ diverges. Since $\nu_{\psi_{1}}=1$ and $\gamma_{\psi_{1}}$ is finite, by Theorem $3, \psi(x)$ converges and the sufficiency proof is complete.

An inequality which $\rho \neq 0$, the radius of convergence of the $\psi$-series, must satisfy, may be easily determined. From the lemma when $\nu_{\psi_{1}}=1, \gamma_{\varphi_{1}} \geqq$ $\gamma_{\psi_{1}}$; also $\left|\varphi_{1}(x)\right|<e^{(1+\epsilon)|x|} e^{\left(\gamma_{\psi_{1}}+\epsilon_{1}\right)|x|},|x|>r_{\epsilon}$. Therefore $\gamma_{\psi_{1}} \leqq 1+\gamma_{\varphi_{1}}$, or $\gamma_{\varphi_{1}}$ $\geqq \gamma_{\psi_{1}} \geqq \gamma_{\varphi_{1}}-1$. Since $\gamma=1 / \rho$, this gives

$$
\rho_{\varphi} \leqq \rho_{\psi} \leqq \frac{\rho_{\varphi}}{1-\rho_{\varphi}}
$$

When $\nu_{\psi_{1}}<1, \mu_{\psi_{1}}>1$ and $\rho_{\psi}$ is infinite, while $\rho_{\varphi} \geqq 1$ for $\gamma_{\varphi_{1}} \leqq 1$. Hence aiways

$$
\rho_{\varphi} \leqq \rho_{\psi} \text { when } \rho_{\psi} \neq 0 \text {. }
$$

According to Theorem 4 the transformation (13) when $\varphi(x)$ is a polynomial beginning with terms of at least the third degree has no invariant analytic curve $y=\psi(x)$ corresponding to the simple root of the cubic (6), though there is obviously a formal $\psi$-series. The curve $y=c x^{2}$ is left invariant when $\varphi(x)=$ $\Sigma_{n=3}^{\alpha}(n-1) x^{n}$, while the curve $y=x^{2} /(1-A x)^{2}$ is left invariant under (13) when $\varphi_{1}(x)=x^{2} e^{A x}\left(e^{x}-1\right)$.

That the number of invariant analytic curves may be infinite is seen from the transformation:

$$
x_{1}=x+x^{2}, \quad y_{1}=y+2 x y+x^{2} y,
$$

which leaves invariant the $\infty^{1}$ curves $y=l_{2} x^{2}, l_{2}$ arbitrary; also from the transformation:

$$
x_{1}=x\left[1+\prod_{j=1}^{m}\left(y-\varphi_{j}(x)\right)\right], y_{1}=y\left[1+\prod_{j=1}^{m}\left(y-\varphi_{j}(x)\right)\right]^{k},
$$

where $\varphi_{j}(x)$ is analytic at $x=0, \varphi(0)=0, m, k$ are positive integers, which leaves invariant the $\infty^{1}$ analytic curves $y=l_{k} x^{k}, l_{k}$ arbitrary and also the $m$ other curves $y=\varphi_{j}(x)(j=1,2, \ldots, m)$. On the other hand the transformation

$$
x_{1}=x+y^{2}, y_{1}=y+x^{3},
$$

possesses not even a formally invariant curve. 
The results obtained in this paragraph show that we can not in general hope to predict the number of power series developments formally invariant under a given transformation, nor, when they exist, can we expect them always to be convergent.

Wellesley College,

WeLLeSLeEY, MAsS. 